\title{
Epigenetic analysis reveals a euchromatic configuration in the FMR1 unmethylated full mutations
}

\author{
Elisabetta Tabolacci ${ }^{1}$, Umberto Moscato ${ }^{2}$, Francesca Zalfa ${ }^{3}$, Claudia Bagni ${ }^{3,4}$, \\ Pietro Chiurazzi ${ }^{1}$ and Giovanni Neri*,1
}

\begin{abstract}
${ }^{1}$ Institute of Medical Genetics, Catholic University, Rome, Italy; ${ }^{2}$ Institute of Hygiene, Catholic University, Rome, Italy; ${ }^{3}$ Department of Experimental Medicine and Biochemical Sciences, Faculty of Medicine, 'Tor Vergata' University, Rome, Italy; ${ }^{4}$ Developmental and Molecular Genetics Section, Faculty of Medicine, Catholic University, Leuven, Belgium
\end{abstract}

Fragile X syndrome (FXS) is caused by the expansion of a CGG repeat in the $5^{\prime}$ UTR of the FMR 1 gene and the subsequent methylation of all $\mathrm{CpG}$ sites in the promoter region. We recently identified, in unrelated FXS families, two rare males with an unmethylated full mutation, that is, with an expanded CGG repeat ( $>200$ triplets) lacking the typical CpG methylation in the FMR1 promoter. These individuals are not mentally retarded and do not appear to be mosaic for premutation or methylated full mutation alleles. We established lymphoblastoid and fibroblast cell lines that showed essentially normal levels of the FMR1mRNA but reduced translational efficiency of the corresponding mRNA. Epigenetic analysis of the FMR1 gene demonstrated the lack of DNA methylation and a methylation pattern of lysines 4 and 27 on histone H3 similar to that of normal controls, in accordance with normal transcription levels and consistent with a euchromatic configuration. On the other hand, histone $\mathrm{H3} / \mathrm{H} 4$ acetylation and lysine 9 methylation on histone $\mathrm{H} 3$ were similar to those of typical FXS cell lines, suggesting that these epigenetic changes are not sufficient for FMR1 gene inactivation. These findings demonstrate remarkable consistency and suggest a common genetic mechanism causing this rare FMR1 epigenotype. The discovery of such a mechanism may be important in view of therapeutic attempts to convert methylated into unmethylated full mutations, restoring the expression of the FMR1 gene.

European Journal of Human Genetics (2008) 16, 1487-1498; doi:10.1038/ejhg.2008.130; published online 16 July 2008

Keywords: fragile X syndrome; unmethylated full mutation; FMR1 gene; epigenetic analysis; DNA methylation

\section{Introduction}

Fragile X syndrome (FXS, OMIM no. 300624) is the most prevalent cause of heritable mental retardation and is caused by the expansion of a polymorphic CGG repeat in the $5^{\prime}$ untranslated (UTR) region of the FMR1 gene. ${ }^{1}$ The

*Correspondence: Dr G Neri, Institute of Medical Genetics, Catholic University, Largo F. Vito 1, Rome 00168, Italy.

Tel: + 39063054 449; Fax: + 39063050 031;

E-mail: gneri@rm.unicatt.it

Received 4 April 2008; revised 17 June 2008; accepted 19 June 2008; published online 16 July 2008
FMR1 gene is involved in the pathogenesis of two other conditions, that is, premature ovarian failure and Fragile $\mathrm{X}$-associated tremor/ataxia syndrome (FXTAS). ${ }^{2,3}$ These two conditions are probably owing to a toxic gain-offunction effect of increased FMR1-mRNA levels. ${ }^{4}$ In FXS patients with a full mutation (with $>200$ CGG repeats), cytosines of the expanded repeat and of the surrounding CpG island of the promoter of the FMR1 gene become methylated. ${ }^{5}$ Although the coding region of the gene remains intact, the combination of these changes, one structural (expansion) and one epigenetic (methylation), results in transcriptional silencing and consequent absence 
of the FMR1 protein (FMRP), which in turn triggers a largely unknown cascade of events, the ultimate effect being the manifestation of the syndrome. ${ }^{6,7}$

The methylation of genomic DNA is catalyzed by DNA methyltransferases (DNMTs), which play a major role in the initiation of chromatin remodeling and gene expression regulation. Until now, three DNMTs have been identified in mammals responsible for DNA methylation during gametogenesis, embryogenesis and somatic tissue development. ${ }^{8}$ A contrasting role of an active DNA demethylase has also been suggested. ${ }^{9}$ However, DNA methylation is not the sole epigenetic change. Several histone modifications, such as acetylation and methylation, are also involved in the regulation of gene expression. Histone acetylation consists of post-translational addition of an acetyl group to the $\varepsilon$-amino group of lysine residues. This reversible modification is catalyzed by several histone acetyltransferases (HATs), classified into three major families. ${ }^{10}$ Histone deacetylation is also an active process, catalyzed by histone deacetylases (HDACs), which are grouped into four classes and two major families. ${ }^{11}$ It is well known that transcription factors and repressor proteins recruit HATs and HDACs to perform sequence-specific actions, acting together as multiprotein complexes. ${ }^{12}$ Non-coding RNAs (ncRNA) may also target these enzymes to specific chromatin regions. ${ }^{13}$ Another post-translational modification occurring on histones is methylation of lysine $(\mathrm{K})$ and arginine $(\mathrm{R})$ residues, catalyzed by histone methyltransferases (HMTs). ${ }^{14}$ Lysine residues can be mono-, di- or trimethylated, and different degrees of methylation of the same residue were shown to differentially affect chromatin structure and transcription. ${ }^{15}$ Although histone methylation was long considered irreversible, the recent identification of numerous site-specific histone demethylases provides compelling evidence that this modification is dynamically regulated. ${ }^{16}$ The methylation of lysine 4 on histone H3 (H3-K4) is a mark of euchromatin or active transcriptional status of the corresponding genes and is established by HMT Set9, associated with coactivator complexes. ${ }^{17}$ On the other hand, methylation of lysine 9 on histone H3 (H3-K9) determines the subsequent recruitment of chromodomain proteins, such as heterochromatin protein 1 (HP1), leading to the formation of heterochromatin. ${ }^{18,19}$ Although heterochromatin formation has become almost synonymous with epigenetic gene silencing, the opposite is also true, at least for some genes, whose expression occurred in the heterochromatic configuration. ${ }^{20,21}$ Several lines of evidence indicate that there might be also an important and conserved role for ncRNAs and RNA interference (RNAi) in the formation and maintenance of constitutive heterochromatin. ${ }^{22}$ The trimethylation of lysine 27 of histone H3 (H3-K27) leads to the recruitment of other Polycomb group proteins and silencing of target genes (heterochromatin). ${ }^{23}$ In contrast, the dimethylated residue is usually associated with both a euchromatic configuration and normally transcribed genes. $^{24}$

In the normally active FMR1 gene, histones $\mathrm{H} 3$ and $\mathrm{H} 4$ are acetylated, H3-K4 is methylated and H3-K9 is hypomethylated, whereas the opposite is true in the transcriptionally inactive methylated full mutation (FXS). ${ }^{25-27}$ Pharmacological treatment of FXS cell lines with HDAC inhibitors (ie, butyrate and TSA) resulted in a modest reactivation of the FMR1 gene, which improved when these drugs were used synergistically with the DNA demethylating agent, 5-azadeoxycytidine (5-azadC). ${ }^{28}$ Consequently, it should be concluded that the critical step in the inactivation of FMR1 is DNA methylation, as also suggested by the existence of rare individuals of normal intelligence who are carriers of an unmethylated full mutation. ${ }^{29}$ The lymphoblastoid cell line 5106, previously characterized by our group, showed absence of DNA methylation in the promoter, essentially normal FMR1mRNA levels and reduced expression and translation efficiency of FMRP. Furthermore, the acetylation status of histones $\mathrm{H} 3$ and $\mathrm{H} 4$ and the methylation of H3-K9 was similar to FXS cell lines, whereas methylation of H3-K4 was like that of normal controls. ${ }^{27}$ In FXS males with a mosaicism of methylated and unmethylated full mutation alleles, FMR1-mRNA levels were found elevated relative to normal controls, suggesting that the CGG expansion per se is not an impediment to transcription. ${ }^{4}$ Elevated FMR1 RNA levels and reduced Fmrp were also described in a mouse model with an unmethylated Fragile $X$ full mutation. ${ }^{30}$ Recently, a human embryonic stem cell (HESC) line was established from a preimplantation FXS embryo, in which DNA was unmethylated and FMR1-mRNA was transcribed despite the presence of a full mutation, whereas epigenetic modifications occurred later, in the course of differentiation. ${ }^{31}$

Here, we report the molecular epigenetic characterization of cell lines established from two newly identified, apparently normal individuals with an unmethylated full mutation, belonging to distinct FXS families. These cell lines showed molecular and epigenetic characteristics similar to those previously observed in the 5106 cell line, ${ }^{27}$ suggesting the existence of a common mechanism as the basis of this rare phenomenon. The discovery of this mechanism may be important in view of therapeutic attempts at converting methylated into unmethylated full mutations, restoring the expression of the FMR1 gene.

\section{Materials and methods}

\section{Cell lines and culture conditions}

Lymphoblastoid cell lines were established by Epstein-Barr virus transformation from peripheral blood lymphocytes of FXS patients, from normal individuals with unmethylated full mutations and from normal control males. The FXS cell lines employed in these experiments are E3, S1 and E6, 
with CGG expansion of 250 (E3), 450 (S1) and 550 (E6) repeats, respectively. ${ }^{32}$ The cell line established from the unmethylated full mutation (code 5106), harbouring an expansion of $\sim 400$ CGG repeats is that established by Smeets et al. ${ }^{29}$ We have recently identified in two distinct FXS families, two apparently normal individuals with an unmethylated full mutation. From each subject (DPM and MA) we established three independent lymphoblastoid cell lines (1, 2 and 3, respectively). A skin fibroblast culture was also obtained from MA. A normal control and FXS fibroblast cultures were also employed. The expansions of the unmethylated full mutation cell lines were in the range of 265-430 CGGs and 430-530 CGGs for MA and DPM, respectively. Lymphoblasts were grown in RPMI-1640 medium and fibroblasts in DMEM medium both supplemented with $10 \%$ fetal bovine serum and penicillin/ streptomycin at $37^{\circ} \mathrm{C}$ with $5 \% \mathrm{CO}_{2}$. Cell medium was changed every $48 \mathrm{~h}$.

\section{Southern blot analysis and bisulphite sequencing}

To check the size of expansion and the methylation status, a double digestion of approx. $10 \mu \mathrm{g}$ of DNA was performed with HindIII and EagI (New England Biolabs). Digested DNA was separated on a $0.8 \%$ agarose gel with $1 \times$ TAE buffer, blotted on Amersham Hybond $\mathrm{N}+$ nylon membrane and hybridized with radioactive Ox1.9 probe. After overnight hybridization and subsequent washing, radioactive filters were exposed to films at $-80^{\circ} \mathrm{C}$ with reinforcing screens before development. The Southern blot analysis was repeated two times on lymphoblastoid cell lines and fibroblasts.

Bisulphite sequencing was performed as previously described by Pietrobono et $\mathrm{al}^{33}$ to analyze the 52 cytosines of the CpG island in the promoter region of the FMR1 gene. For each cell line (wild-type, FXS and unmethylated full mutations) seven different clones were sequenced.

\section{Quantitative RT-PCR analysis}

Total RNA was extracted with the single-step acid phenol method using Trizol (Invitrogen). Four independent RNA extractions were performed from lymphoblastoid cell lines established from three different normal controls, three FXS and three replicates each from DPM and MA, respectively. Five independent RNA extractions were performed for fibroblast cultures established from one normal control, one FXS and from MA. RNA was reverse-transcribed by MoMLV-reverse transcriptase (Invitrogen). For a quantitative estimate of the FMR1-mRNA levels, we adapted the technique described by Tassone et al, ${ }^{4}$ using an ABI 7900 Sequence Detector with dual-labelled TaqMan probes. The FMR1 amplicon is a 89-bp product spanning the junction between exon 13 and 14 of the gene (positions 1432-1520 of GenBank sequence NM_002024). The primers and TaqMan probe employed were as described by Pietrobono et al. ${ }^{33}$ The relative amount of FMR1-mRNA was assessed by comparison with the human HPRT-mRNA detected with the Pre-Developed TaqMan Assay Reagent (ABI 4310890E: huHPRT endogenous control).

\section{Western blotting analysis}

Proteins extracted from normal, FXS, DPM and MA cell lines (both lymphoblasts and fibroblasts) were resuspended in Laemli buffer, boiled, separated on $8 \%$ polyacrylamide gel electrophoresis, transferred to Immobilon-P membrane (Millipore), immunostained and visualized using the ECL Western Blotting Kit (GE Healthcare), according to the manufacturer. Primary antibodies were used at the following concentrations: 1:500 anti-FMRP mouse monoclonal antibody T1a (Società Italiana Chimici) and 1:1000 antitubulin mouse antibody (Sigma-Aldrich). Two separate blots were hybridized with anti-tubulin antibody and two additional independent blots were also hybridized with antiribosomal protein S6 polyclonal antibody (Società Italiana Chimici). Protein quantification was obtained by densitometric measurements of the signal intensity after film exposure (ECL).

\section{Polysomes/mRNPs distribution analysis}

Each gradient from wild-type, FXS, DPM and MA cell lines (both lymphoblasts and fibroblasts) was collected in 10 fractions, monitoring the optical density at $254 \mathrm{~nm}$ as described by Pietrobono et al. ${ }^{27}$ RNA was extracted from each gradient fraction using the proteinase $\mathrm{K}$ method and DNase treatment. Equal amounts of RNA from fractions 1 to 5 were pooled and designated fraction $\mathrm{A}$, which represents RNA binding to polysomes; the pool from fractions 6 to 10 (fraction B) represents RNA binding to monosomes (mRNPs). The two pools were reverse transcribed into cDNA by MoMLV-reverse transcriptase (Invitrogen) for three times and analyzed in three independent quantitative reverse transcriptase (RT)-PCR using ABI7900 Sequence Detector with dual-labelled TaqMan probes. The relative amount of FMR1-mRNA was measured by comparison with the human HPRT-mRNA detected with the Pre-Developed TaqMan Assay Reagent (ABI 4310890E: huHPRT endogenous control), as previously described. A second endogenous control, human ribosomal protein S6 (Hs02339423_g1), was also employed. Relative mRNA levels were calculated as previously described.

\section{Chromatin immunoprecipitation assay}

We employed the chromatin immunoprecipitation assay (ChIP) Assay Kit and antibodies from Upstate Biotechnology (NY, USA) and followed the manufacturer's protocol. Histone acetylation was studied using antibodies directed against histone $\mathrm{H} 4$ acetylated at lysines 5, 8, 12 and 16 (17-229), respectively, and against histone $\mathrm{H} 3$ acetylated at lysines 9 and 14 (17-245). Histone methylation analysis was performed using two different antibodies against histone H3, dimethylated at lysine 9 (H3-K9, 07-441) or 
at lysine 4 (H3-K4, 07-030). The methylation status of lysine 27 on histone H3 was studied with two different antibodies against dimethylated (diH3-K27, 07-452) and trimethylated (triH3-K27, 07-449) forms, respectively. After chromatin immunoprecipitation and prior to quantitative fluorescent PCR analysis, DNA was extracted with a standard procedure (phenol/chloroform/isoamilic alcohol 25:24:1), checked spectrophotometrically and on agarose gel and digested with XhoI, to separate the CGG repeats that could interfere with the amplification efficiency.

\section{Quantification of immunoprecipitated DNA}

The levels of acetylation of histone $\mathrm{H} 3$ and $\mathrm{H} 4$ and of methylation of H3-K9, H3-K4 and H3-K27 of both FMR1 and HPRT were measured by fluorescent PCR with the ABI 7700 Sequence Detector (Applied Biosystems) with dual labelled TaqMan probes. Primers and probes employed for PCR analysis were designed to amplify three different portions of the FMR1 gene: the promoter region (positions 13376-13448 of GenBank L29074), exon 1 near the CGG repeat tract (positions 13940-14012 of GenBank L29074) and exon 16 (positions 47 305-47 386 of GenBank L29074). The HPRT amplicon overlapped exon 6 (positions 34946-35022 of GenBank sequence M26434). The sequences of primers and probes were described in detail by Pietrobono et al (2005). A standard curve for the three FMR1 and for the single HPRT amplicon was constructed with five different DNA dilutions of known concentration $(X$ axis $=\log [\mathrm{X}])$ and the corresponding $C_{\mathrm{t}}$ values ( $Y$ axis). The unknown amount of acetylated and methylated immunoprecipitated DNA (IP-DNA) of FMR1 and HPRT $\left(X\right.$ axis $=\log [\mathrm{X}]$ ) was calculated from $C_{\mathrm{t}}$ values, through the standard curve plot. Normalized FMR1 levels were estimated dividing the amount of FMR1 IP-DNA by the amount of HPRT IP-DNA. The acetylation and methylation levels of DPM and MA lymphoblastoid cell lines were compared with the levels of two different FXS and control cell lines, whereas for MA fibroblasts one FXS and one control cell line was employed. Two independent ChIPs with anti-H3 and - $\mathrm{H} 4$ and anti-H3-K4 and -H3-K9 antibodies were performed for each lymphoblastoid cell line and three for fibroblast cell lines, whereas three independent ChIPs with anti diH3-K27 and triH3-K27 antibodies were carried out for all cell lines analyzed.

\section{Statistical analysis}

All variables were analyzed by means of descriptive statistics (mean, standard deviation, standard error and confidence intervals of mean). The 'Student's' $t$-test (twosample mean comparison test) was employed to explain the total variation, evaluating the ratio between the mean values of wild-type (wt) vs methylated full mutations (FXS) and/or $v s$ unmethylated full mutations (DPM and MA).

We tested the 'null hypothesis' $\mathrm{H}_{0}$ that no real difference of variation existed in histone acetylation and methylation levels between wt and DPM or MA vs FXS in the three regions of the FMR1 gene analyzed. If the ratio between the mean values ( $t$-test) of both wt and DPM/MA vs FXS was near \pm 1 , the null hypothesis $H_{0}$ was accepted; whereas if $t$-test was $\neq 1$, wt and DPM/MA were independent variables with respect to FXS and consequently the 'null hypothesis' $\mathrm{H}_{0}$ was rejected and the 'alternative hypothesis' $\mathrm{H}_{\mathrm{A}}$ was accepted. The test was two-tailed and the level of significance $P$ was $\leq 0.01$. The collection and analysis of data were performed using STATA Intercooled version 9.2 software for MacIntosh (Stata Co., College Station, Lakewag, TX, USA).

\section{Results \\ Clinical and molecular description}

We have recently identified, in two distinct FXS families, two apparently normal males (DPM and MA) with an unmethylated full mutation in their peripheral blood leukocytes. DPM was 34 and MA 33 years old. They were referred for genetic testing, being maternal uncles of two FXS boys. In particular, MA belongs to the family described by Tabolacci et al, ${ }^{34}$ in which his sister had three sons, two affected and one normal. The latter was carrier of a nonmosaic, apparently normal FMR1 allele that resulted from the reduction of the expanded maternal allele. Physical and psychomotor development of both DPM and MA was apparently normal, even though they refused formal IQ testing. They had a normal school record, a steady job and conducted independent lives. DPM is married but has no children. From each individual we established three independent lymphoblastoid cell lines, to check the possibility that different cell lines from the same individual may represent different clones, with varying levels of CGG expansion and/or methylation. A fibroblast cell line was also obtained from MA, with the purpose of studying another tissue, different from blood.

We repeatedly checked DNA extracted from cell cultures by Southern blotting after double digestion with HindIII and EagI (Figure 1a). A single unmethylated band was seen at $3.2 \mathrm{~kb}$, corresponding to a $0.7 \mathrm{~kb}$ expansion or approximately 265 CGG repeats for MA fibroblasts. The three MA lymphoblastoid cell lines showed bands in a range from 3.2 to $3.7 \mathrm{~kb}$, corresponding to unmethylated full mutation alleles of $0.7-1.2 \mathrm{~kb}(265-430$ CGGs). The three DPM lymphoblastoid cell lines had unmethylated alleles ranging from 3.7 to $4.0 \mathrm{~kb}$, equivalent to $1.2-1.5 \mathrm{~kb}$ expansions (430-530 CGGs). The lymphoblastoid cell lines reflected the blood DNA status of these individuals. We repeated Southern blot analysis two times. As to the methylation status of each CpG site in the FMR1 promoter region, seven independent clones of each cell line were found to be predominantly unmethylated as in a wild-type, opposite to a FXS, whose $52 \mathrm{CpG}$ residues were all methylated (Figure 1b). 


\section{a}

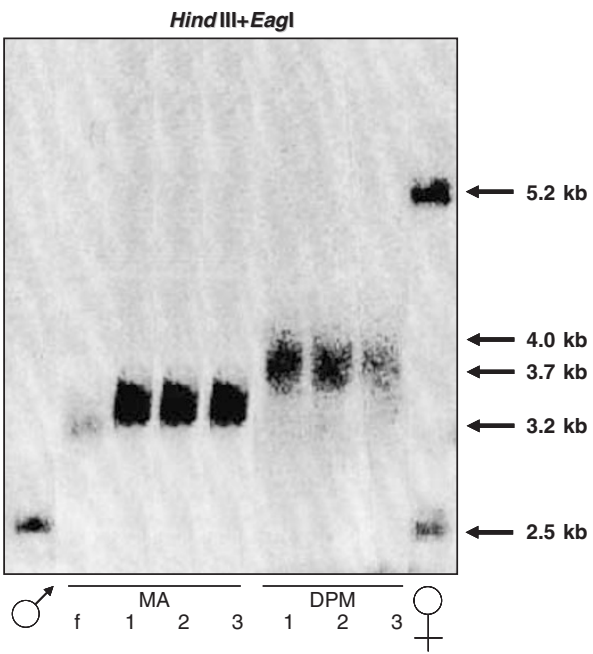

b
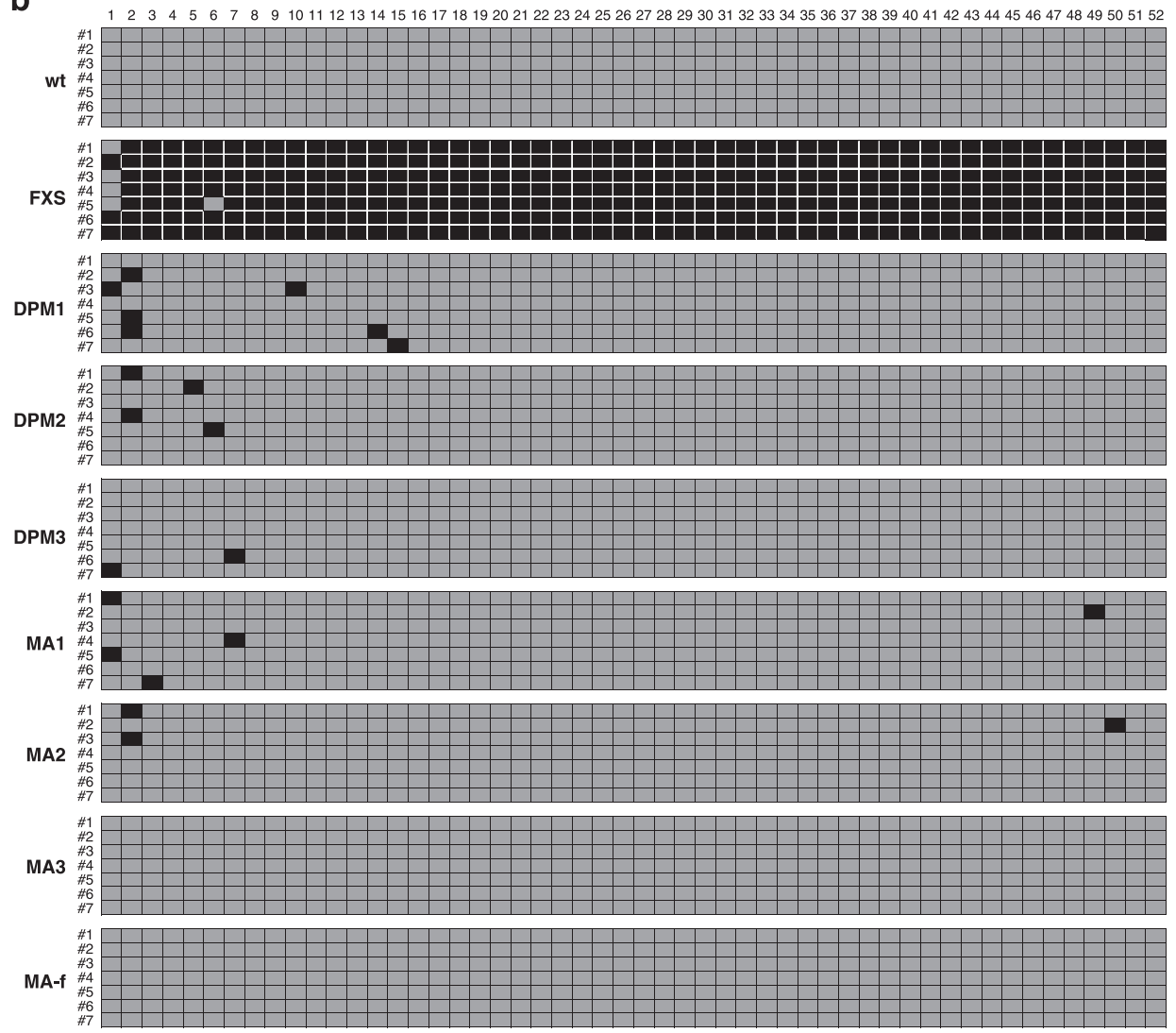

Figure 1 Southern blotting analysis and bisulphite sequencing of the FMR1 promoter region. (a) Southern blot using the Ox1.9 radioactive probe on a double digestion with restriction enzymes HindllI and Eagl to assess the size and methylation status of the cell lines. A normal male (lane 1) shows a $2.5 \mathrm{~kb}$ band, whereas a normal female (lane 9) shows an additional band at $5.2 \mathrm{~kb}$, corresponding to the methylated FMR1 gene on the inactive $\mathrm{X}$ chromosome. MA fibroblasts (lane 2) display a single band at $3.2 \mathrm{~kb}$, corresponding to an unmethylated full mutation of approximately 235 CGG repeats; the three MA lymphoblastoid cell lines (lanes 3-5) show bands from 3.2 to $3.7 \mathrm{~kb}$, corresponding to an unmethylated full mutation of 265-430 CGGs. The three DPM lymphoblastoid cell lines (lanes 6-8) have bands from 3.7 to $4.0 \mathrm{~kb}$, equivalent to an unmethylated full mutation of 430-530 CGG repeats. (b) FMR1 promoter region of normal control male (top), methylated full mutation (FXS, middle) and unmethylated full mutation allele (DPM1-3, MA1-3 and MA-f). Every line corresponds to bisulphite sequencing of an individual cell. For each cell line, seven different cells are sequenced. From left to right, black or grey square correspond to the 52 methylated or unmethylated CpG sites, respectively. After bisulphite treatment the cytosines of the $\mathrm{CpC}$ sites were transformed into thymines in the unmethylated full mutation like in the normal control male, whereas in the FXS they remain unmodified. 
We then quantified the FMR1-mRNA levels in all unmethylated full mutations, FXS and normal control cell lines by real-time RT-PCR (Figure 2). Four different measurements were obtained from four separate RNA extractions. No FMR1-specific mRNA was detected in FXS cell lines. With respect to three different normal control cell lines, the unmethylated full mutation lymphoblastoid cell lines had essentially normal levels of FMR1-mRNA (range 0.7-1.3, compared with the control value, arbitrarily set at 1.0 ) as shown in Figure 2 a. Five separate RNA extractions and quantitative RT-PCR were performed from fibroblasts, showing that the FMR1-mRNA levels of MA were on average 1.5-fold higher than those of a normal control male (Figure 2b).

Figure 3 shows the FMRP levels evaluated by Western blotting analysis. Four separate Western blots were performed and probed with anti-FMRP mouse monoclonal antibody T1. Protein levels were normalized with respect to tubulin and to rpS6 (not shown). The amount of FMRP in all unmethylated full mutations was estimated to be on average $20 \%$ compared with a normal control cell line and normalized to tubulin (in two independent experiments).

Finally, to confirm the reduced translational efficiency, already observed in the 5106 cell line by Pietrobono et al (2005), we performed polysome/mRNPs distribution analysis (Figure 4). The results from lymphoblastoid cell lines are reported in Figure $4 \mathrm{a}$ and those from MA fibroblasts in Figure $4 \mathrm{~b}$. In both types of cells, FXS lines showed absence of specific FMR1-mRNA and presence of endogenous control mRNAs (HPRT and rpS6) binding to polysomes and to monosomes, as expected. On the other hand, in cell lines with an unmethylated full mutation the FMR1-mRNA was lower in fraction A (polysomes) compared with fraction B (monosomes), whereas rpS6-mRNA
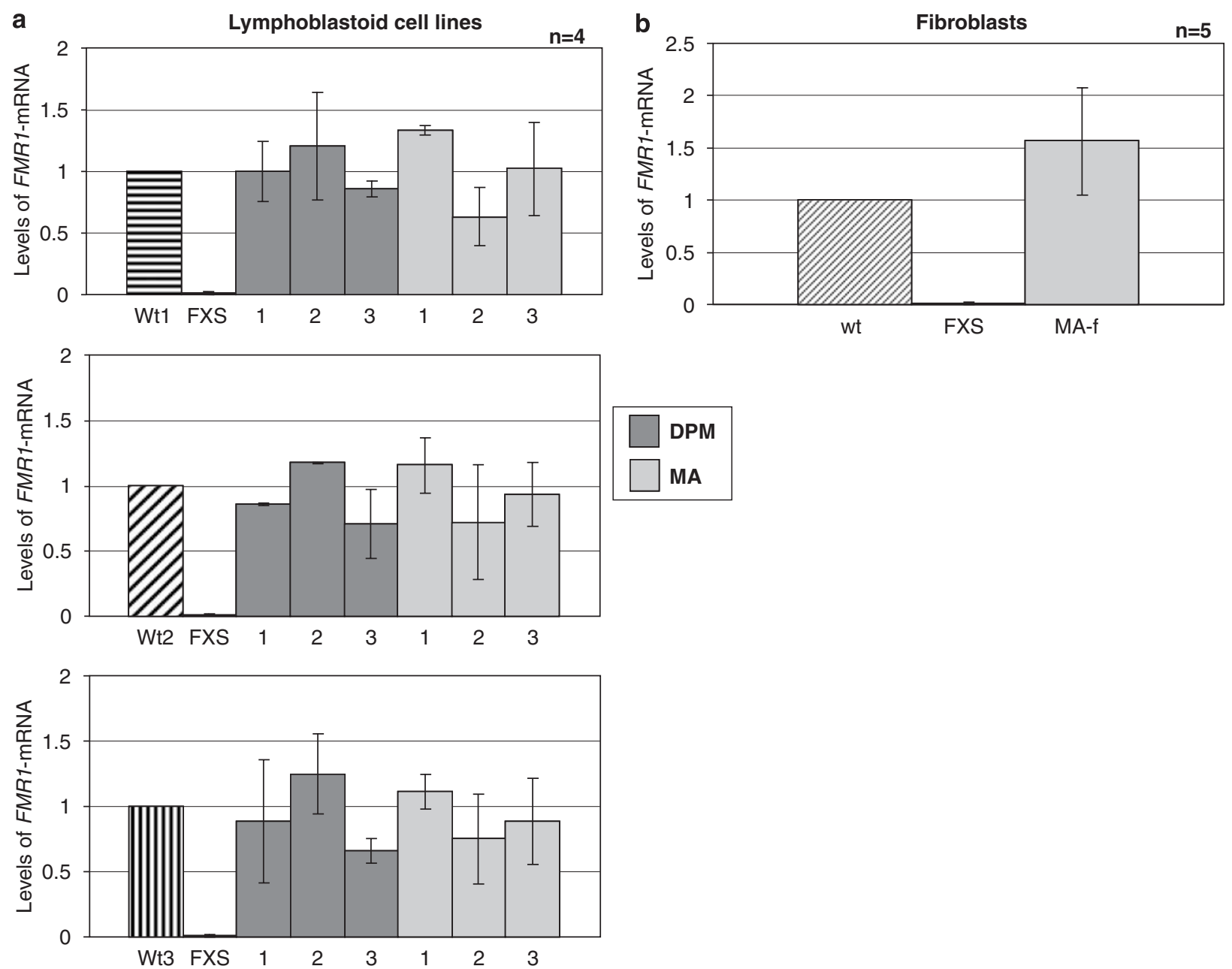

Figure 2 Quantitative estimate of FMR1-mRNA levels (mean \pm SD) in the three unmethylated full mutations and in an FXS by real-time RT-PCR, normalized to three different normal control cell lines (wt1-3) (a). Panel b shows the FMR1-mRNA quantification in MA and FXS fibroblasts, compared with a normal control cell line. Note that a total of four independent RNA extractions and RT-PCRs were performed for lymphoblastoid cell lines and five for fibroblasts. 

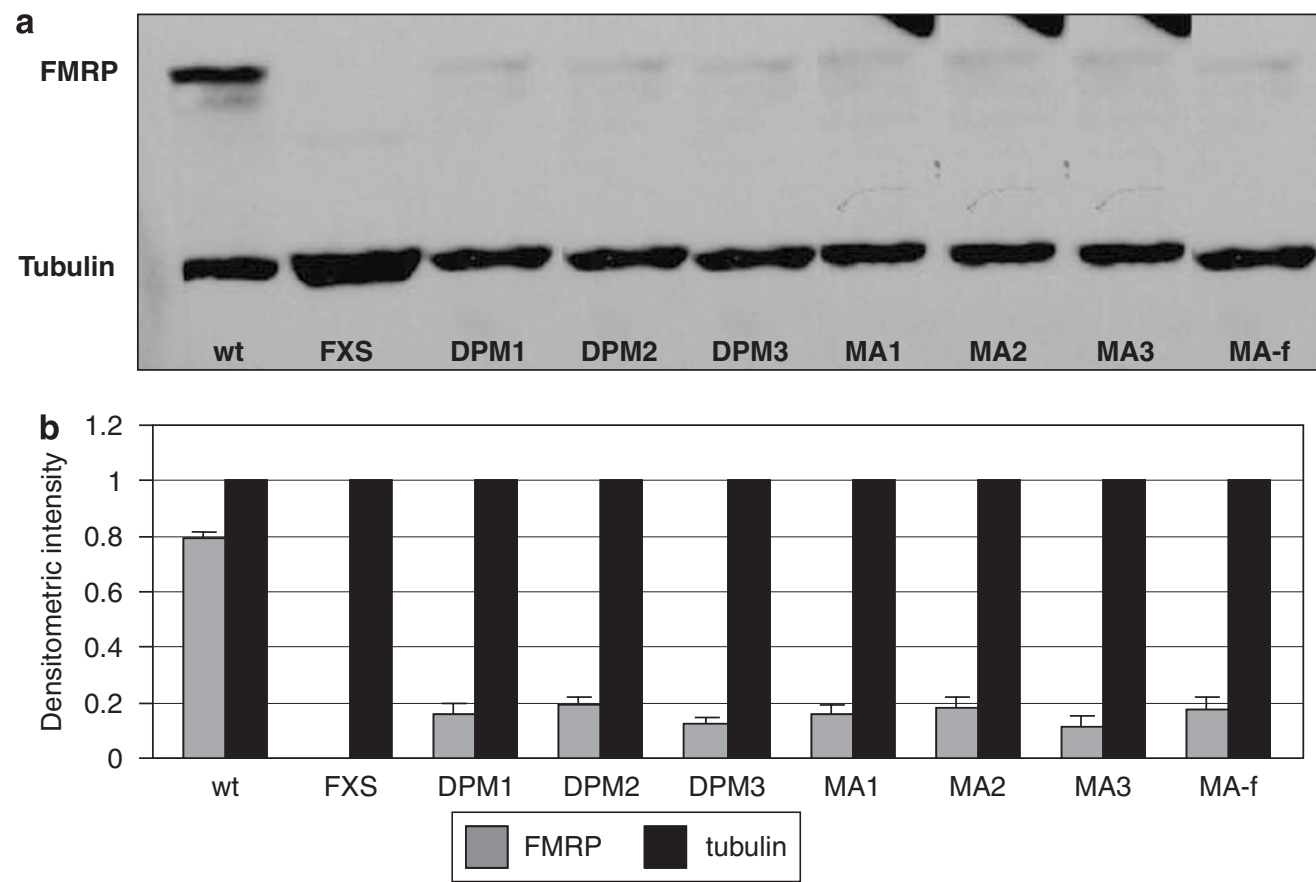

Figure 3 Western blotting analysis of FMRP, visualized with ECL kit. Protein extracts from normal control (wt), FXS, DPM1-3, MA1-3 and MA fibroblasts were loaded and probed with an anti-FMRP mouse monoclonal T1a antibody and one against the tubulin (top). Relative protein levels corresponding to FMRP were normalized to tubulin in the control, FXS and unmethylated full mutation cell lines (bottom). The amount of FMRP in all unmethylated full mutations is estimated to be around $20 \%$ compared with a normal control cell line (on two independent blots).
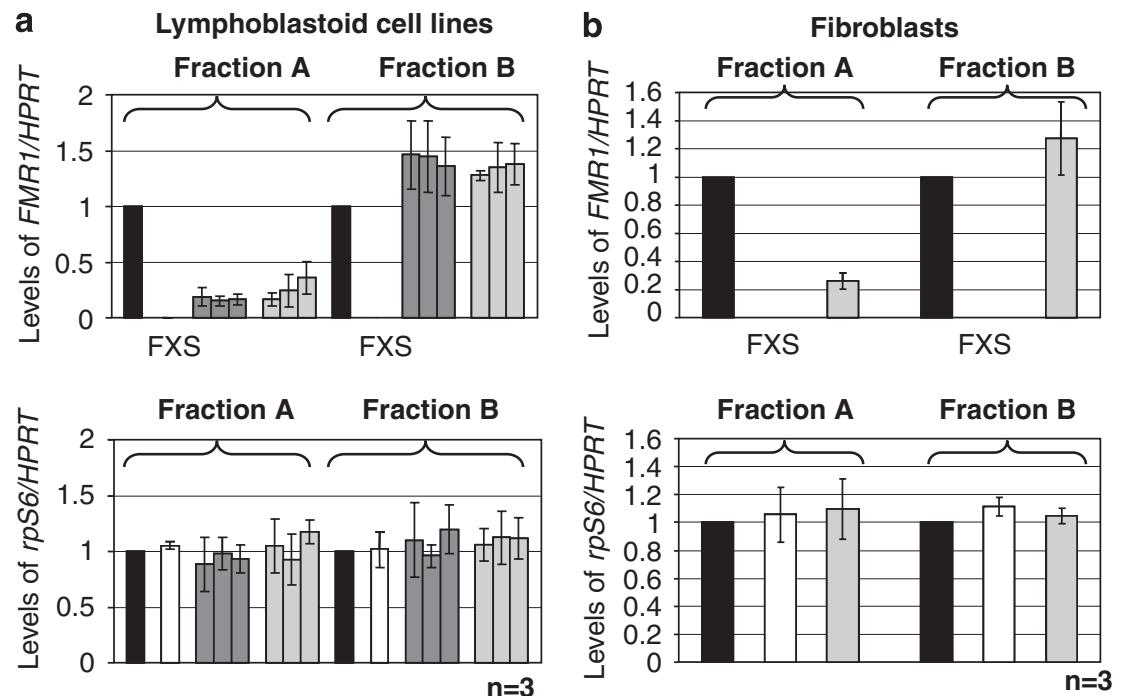

wt $\square$ FXS $\square$ DPM $\square$ MA

Figure 4 Quantitative RT-PCR analysis (mean \pm SD) of gradient fractions with primers specific for $r p S 6$ (the transcript for ribosomal protein S6), FMR 1 and HPRT. Equal amount of RNA from fraction 1 to 5 were pooled (fraction A), representing RNA-binding to polysomes; the pool from fraction 6 to 10 (fraction B) represented RNA-binding to monosomes (mRNPs). The two pools were reverse transcribed into cDNA for three times and analyzed in three independent quantitative RT-PCRs. The relative amounts of FMR1-mRNA were estimated by comparison with the human HPRT and rpS6 mRNAs and normalized to a normal control cell line. The results from lymphoblastoid cell lines are in panel $\mathbf{a}$ and those from fibroblasts in panel $\mathbf{b}$.

was equally represented in both fractions. These data indicate a reduced translation efficiency of the expanded FMR1-mRNA compared with wild type (around 30\%).

\section{Epigenetic characterization}

Acetylation levels of histone $\mathrm{H} 3$ and $\mathrm{H} 4$ and methylation levels of histone H3 lysine 4 and 9 in the promoter 

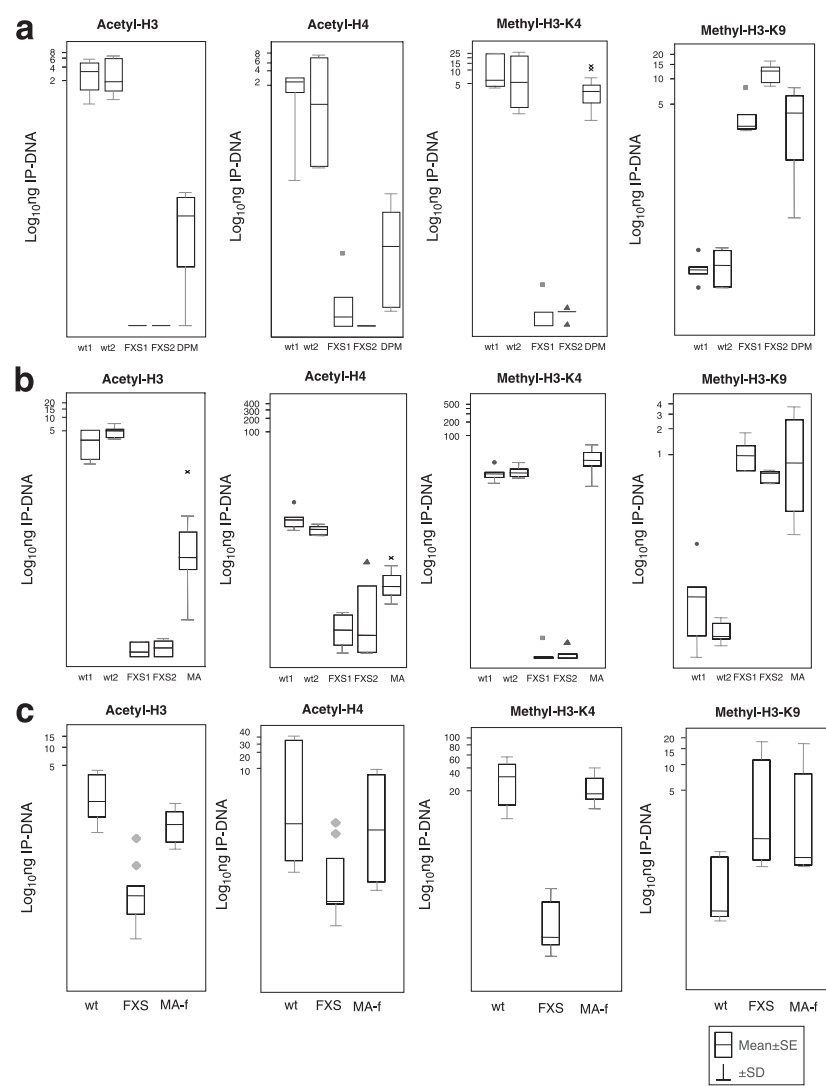

Figure 5 ChIP analysis of histone $\mathrm{H} 3$ and $\mathrm{H} 4$ acetylation and histone $\mathrm{H} 3-\mathrm{K} 4$ and $\mathrm{H} 3-\mathrm{K} 9$ methylation of the promoter region of the FMR1 gene in DPM (a) and MA (b) lymphoblasts, and in MA fibroblasts (c). The corresponding histone acetylation and methylation levels of two different normal control (wt1-2) and FXS (FXS1-2) cell lines are also reported. Box-plots indicate the mean of two independent immunoprecipitations for each lymphoblastoid cell lines (DPM1-3 and $\mathrm{MA} 1-3$ ) and three for fibroblasts and the corresponding standard error and standard deviation (not reported in those instances where it is approximately zero). Note that the amount of IP-DNA (ng) is indicated in logarithmic scale.

region of the FMR1 gene are reported in Figure 5. The epigenotype was significantly different between wildtype and FXS cell lines, as previously described. ${ }^{27}$ Wild-type cell lines (wt1 and wt2) showed higher H3 and H4 acetylation ( $t=4.0114$ and 4.3512 , respectively), higher H3-K4 methylation $(t=6.1672)$ and lower H3-K9 methylation $(t=-5.3621)$ levels compared with FXS cell lines (FXS1 and FXS2). Similar epigenetic changes were also observed in FMR1 exon 1 in both cell types (lymphoblasts and fibroblasts), whereas in exon 16 the epigenetic differences were minimal (see Supplementary Figure S1). In unmethylated full mutation lymphoblastoid cell lines, histone $\mathrm{H} 3$ and $\mathrm{H} 4$ acetylation levels were low, similar to those of FXS cell lines. Similarly, histone H3-K9 methylation was more similar to that of the two FXS lines ( $t=-3.1228$ and -2.4412 for DPM and MA, respectively). On the other hand, H3-K4 methylation was high, like in normal control cells $(t=2.8883)$ (Figure $5 \mathrm{a}$ and $\mathrm{b}$ ). The MA fibroblasts showed the same epigenetic pattern as the corresponding lymphoblasts, with the exception of $\mathrm{H} 3 \quad(t=2.6717)$ and $\mathrm{H} 4$ acetylation levels, which were similar to those of normal cells (Figure 5c). All the above-mentioned changes were also observed in the exon 1 region, and to a lesser degree in exon 16 (see Supplementary Figure S1).

The methylation status of histone H3 lysine 27 (K27) in the promoter region of FMR1 is shown in Figure 6. Both lymphoblastoid (panel a) and fibroblast cell lines (panel b) of unmethylated full mutation carriers had lower levels of trimethylation and higher levels of dimethylation compared with FXS cell lines. This epigenetic condition was similar to that of normal control cell lines and consistent with a euchromatic configuration. The same epigenetic pattern observed in the promoter region was found in exon 1 and only partially in exon 16 (see Supplementary Figure S2). The $t$ values (with $P \leq 0.01$ ) of FXS $v s$ unmethylated full mutations of dimethyl- and trimethyl-H3-K27 in both lymphoblastoid cell lines and fibroblasts for the three regions of the FMR1 gene analyzed are reported in Table 1. The 5106 cell line, previously not characterized for lysine 27, exhibited the same pattern as the DPM and MA unmethylated full mutations, as one could have expected (data not shown).

\section{Discussion}

The critical step in the inactivation of the FMR1 gene, consequent to the expansion of the CGG triplets above the threshold of 200 repeats and triggering the cascade of events that lead to the expression of the FXS phenotype, appears to reside in the epigenetic changes that affect histones and DNA in the promoter and exon 1 region. ${ }^{27}$ Therefore, understanding the molecular mechanisms responsible for these epigenetic changes is critical for a full comprehension of the pathophysiology of FXS. Actually, nature provides us with a very useful model, that is, those rare individuals who, for as yet unclear reasons, fail to methylate the FMR1 full mutation. This failure rescues them from developing the FXS phenotype, a phenomenon that could have practical implications for the treatment of fragile $\mathrm{X}$ patients. The present report aims at clarifying the nature of this phenomenon.

The molecular characterization of the unmethylated full mutations from two rare individuals of normal intelligence showed absence of DNA methylation both in the promoter region and in the expanded CGG tract of the FMR1 gene. No evidence of mosaicism with methylated alleles was present in either peripheral leukocytes or in lymphoblastoid and fibroblast cell lines. The Southern blotting analysis was repeated several times and no evidence of instability was found, in contrast to the observations of Whorle et al. ${ }^{35}$ 


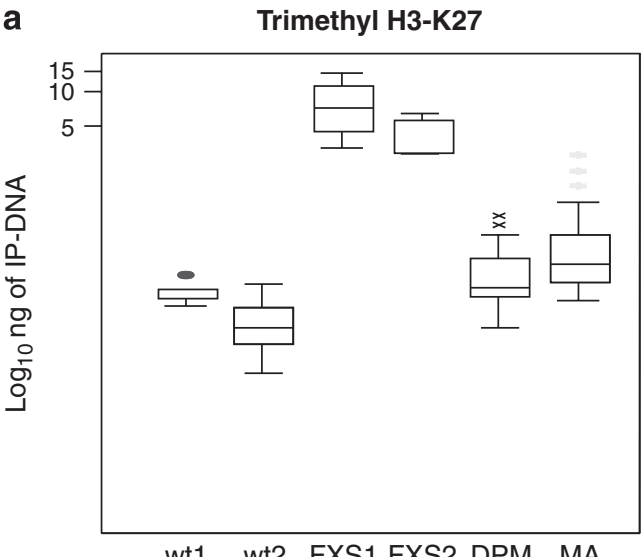

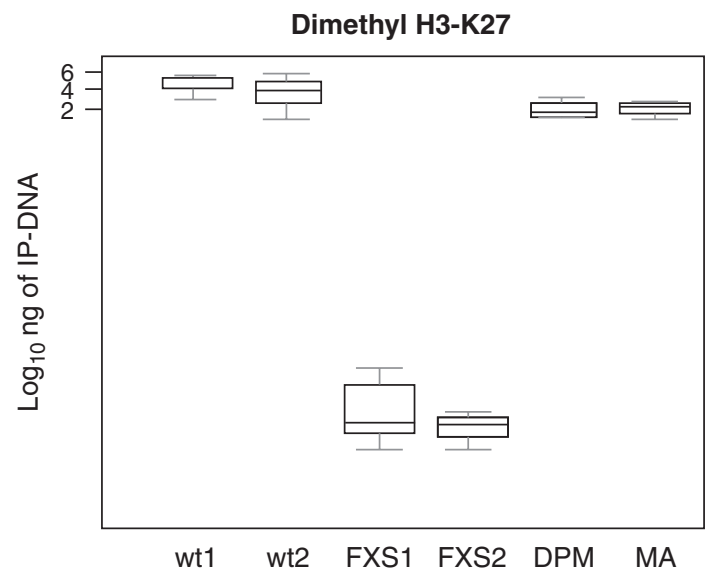

b Trimethyl H3-K27

Dimethyl H3-K27
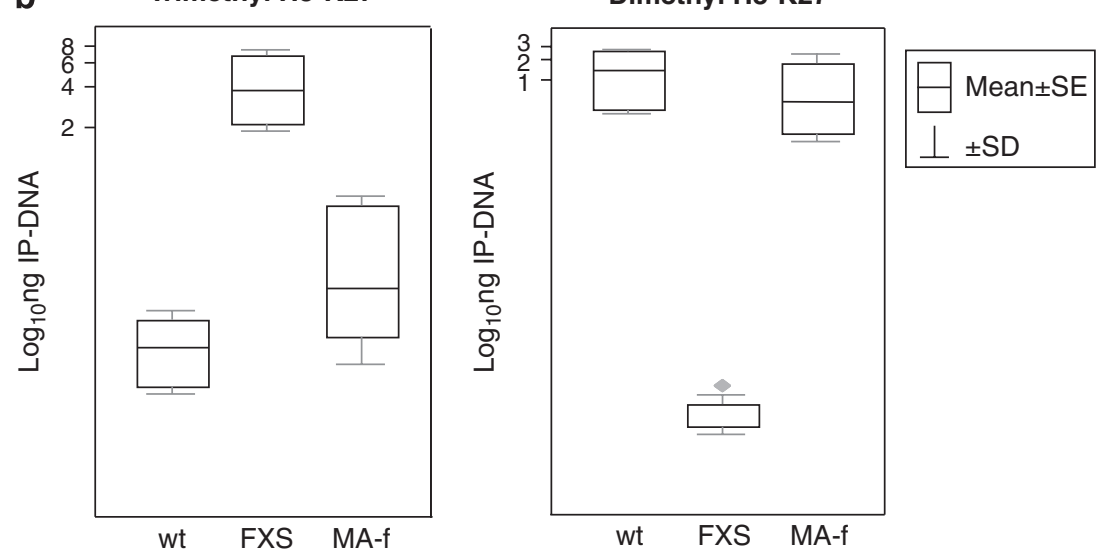

Figure 6 ChIP analysis of histone H3-K27 di- and trimethylation of the promoter region of the FMR1 gene in DPM and MA lymphoblasts (a) and MA fibroblasts (b). The corresponding H3-K27 di- and trimethylation levels of two different normal control (wt1-2) and FXS (FXS1-2) cell lines are also reported. Box-plots indicate the mean of three independent immunoprecipitations both for lymphoblasts and for fibroblasts and the corresponding SE and SD (not reported in those instances where it is approximately zero). Note that the amount of IP-DNA (ng) is indicated in logarithmic scale.

Table 1 'Student' $t$ values of FXS vs unmethylated full mutations of dimethyl- and trimethyl-H3-K27 in both lymphoblastoid cell lines and in fibroblasts for the three regions of the FMR1 gene analyzed

\begin{tabular}{llc}
\hline Dimethyl H3-K27 & Lymphoblastoid cell lines & Trimethyl H3-K27 \\
\hline$t=-3.0626$ & FXS vs DPM promoter & $t=8.9413$ \\
$t=-2.9936$ & FXS vs MA promoter & $t=8.2662$ \\
$t=-2.4435$ & FXS vs DPM exon 1 & $t=5.3201$ \\
$t=-2.8479$ & FXS vs MA exon 1 & $t=3.0306$ \\
$t=-2.2033$ & FXS vs DPM exon 16 & $t=5.3007$ \\
$t=-1.8770$ & FXS vs MA exon 16 & $t=4.9850$ \\
& & \\
& Fibroblasts & $t=3.6131$ \\
$t=-2.8957$ & FXS vs MA promoter & $t=2.4715$ \\
$t=-4.2286$ & FXS vs MA exon1 & $t=4.8596$ \\
$t=-2.0699$ & FXS vs MA exon 16
\end{tabular}

The level of significance $P$ is $\leq 0.01$ for all $t$-values.

FMR1 transcription was essentially normal in lymphoblasts and slightly elevated in fibroblasts, compared with appropriate normal controls. This observation appears to be in contrast with the deduction of Tassone et al, ${ }^{36}$ claiming that mosaic males with methylated and unmethylated full mutations have very high levels of FMR1mRNA, similar to premutation carriers. ${ }^{4}$ This may be because of the different cell types investigated, given that Tassone et $a l^{36}$ measured mRNA in fresh white blood cells whereas we used either lymphoblastoid cell lines or fibroblasts in culture. The presence of elevated levels of FMR1-mRNA appears to have a toxic 'gain-of-function' effect in premutation carriers, ${ }^{3}$ who may develop a neurodegenerative disorder (tremor-ataxia syndrome or FXTAS). It is difficult to predict whether carriers of unmethylated full mutations have a higher risk of developing FXTAS such as premutation males. In fact, they do express FMR1-mRNA with an expanded CGG tract, although our data suggest that mRNA levels may be lower than those estimated by Tassone et al. ${ }^{36}$

In any case, our findings confirm that the CGG expansion per se does not prevent transcription, whereas the presence of a longer CGG tract may reduce the 
translational efficiency, ${ }^{37,38}$ as demonstrated by our results with polysomes/mRNPs profiling and with Western blotting (reduced FMRP levels). Recently, it was demonstrated that secondary structures of expanded CGG repeats in
mRNA obstruct its translation, whereas quadruplexdisrupting proteins alleviate the translational block. ${ }^{39}$

Unless some neurons in the brain of these individuals harbour a premutation, which we cannot rigorously
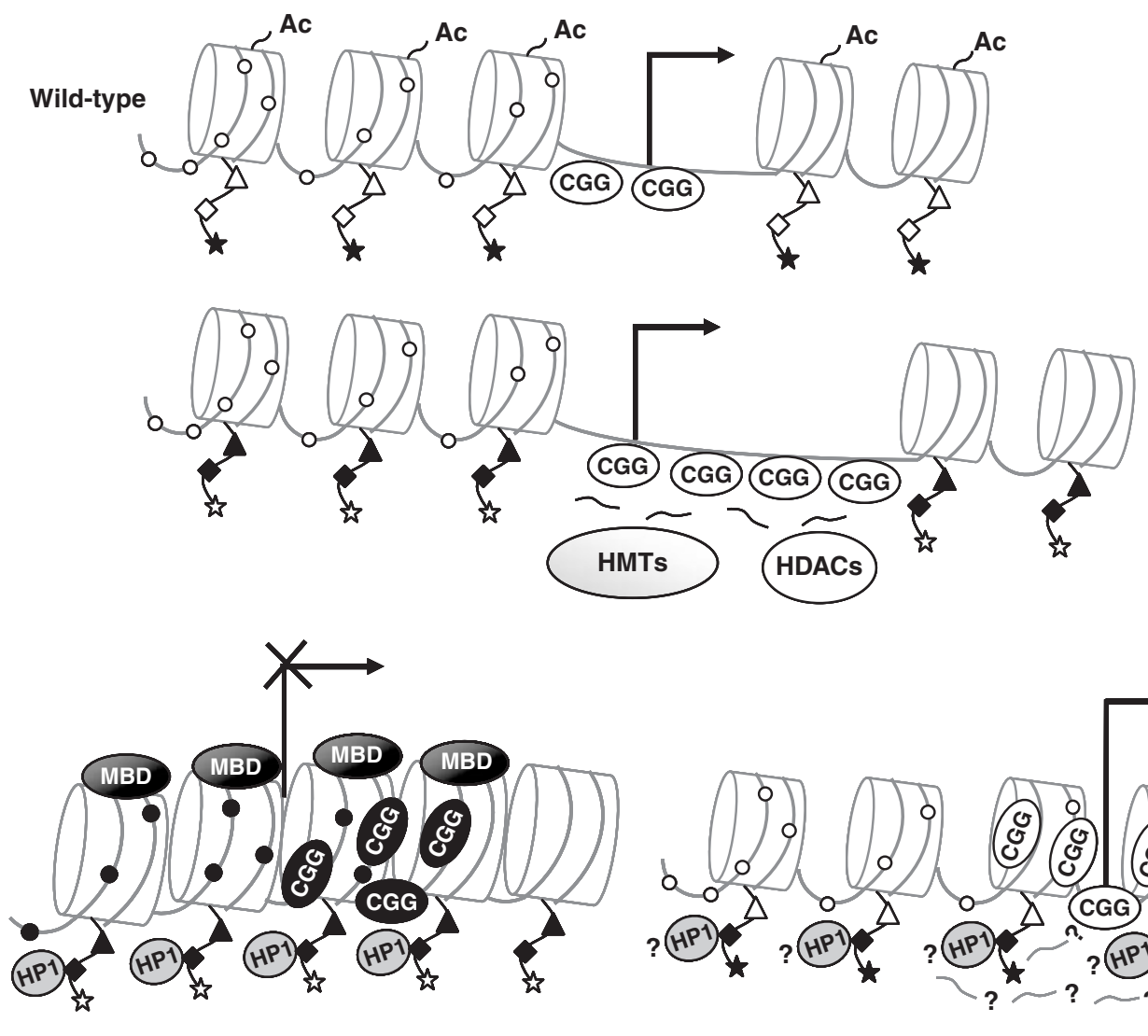

Methylated full mutation

(FXS)

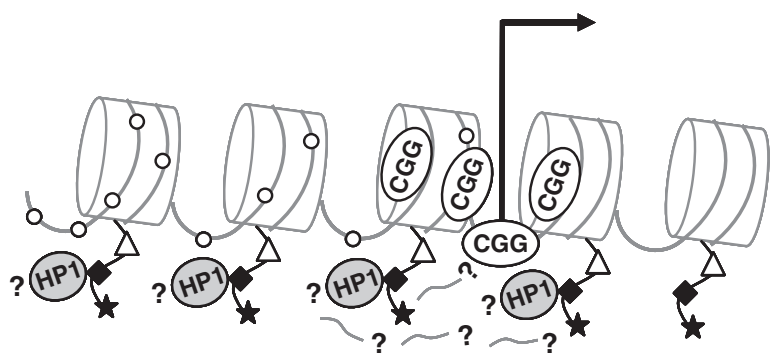

Unmethylated full mutation

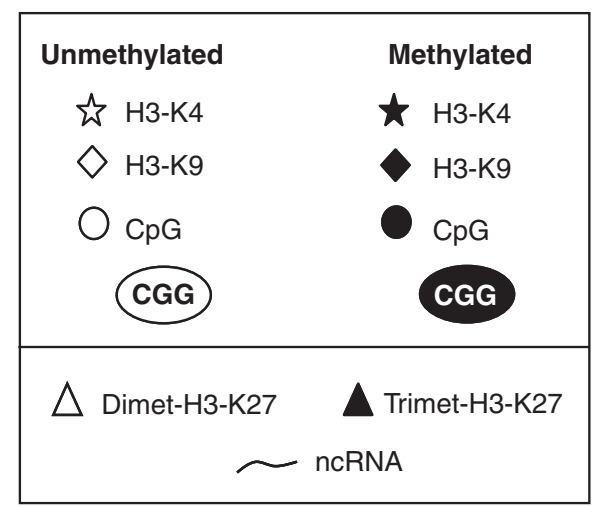

Figure 7 Schematic representation of the epigenetic marks in the $5^{\prime}$-UTR of the FMR1 gene. In the wild type the cytosines of the CpG island of the promoter region and of the CGG sequence are not methylated, histones $\mathrm{H} 3$ and $\mathrm{H} 4$ are acetylated, histone $\mathrm{H} 3$ tails are methylated only at lysine (K) 4 , and lysine 27 on histone $\mathrm{H} 3$ is dimethylated. The FMR1 gene is normally transcribed (arrow). In FXS, when the CGG sequence expands over 200 triplets, during the early stages of embryogenesis, the DNA methylation is absent and the FMR1 transcription is normal, probably in the presence of ncRNA-specific sequences, which recruit histone methyltransferases (HMTs) and deacetylases (HDACs), establishing the epigenetic marks. Then DNA methylation maintains the silent status of the gene through methyl-binding proteins (MBDs) and heterochromatin protein 1 (HP1). The unmethylated full mutations may have abnormal/absent production of ncRNAs specific for the FMR1 gene. This supposed defect would result in lack of recruitment of HMTs and heterochromatin formation, justifying the presence of normal transcription. 
exclude, it would seem that reduced levels of FMRP, not exceeding $30 \%$ of normal, are sufficient for normal intellectual functioning. Tissue heterogeneity of FMR1 gene expression was described in a 'high-functioning' FXS male, who was a mosaic of methylated and unmethylated full mutation. Lack of mental retardation in this individual may have been because of sufficient expression of FMRP in most areas of the brain. ${ }^{40}$

The epigenetic pattern of the lymphoblastoid cell lines obtained from DPM and MA was similar to that previously observed by us in the 5106 cell line. ${ }^{27}$ This pattern reflects that of normal control cells, with the exception of the low $\mathrm{H} 3$ and $\mathrm{H} 4$ acetylation levels, which was more similar to that of FXS cells. Surprisingly, fibroblasts from MA had higher H3 and $\mathrm{H} 4$ acetylation levels, similar to those of normal controls. This slightly different epigenetic status may be because of the difference in cell type and may explain the increased level of FMR1-mRNA observed in MA fibroblasts, compared with a normal control. Importantly, histone H3K4 methylation level was consistently high, similar to that of a normal control, in accordance with normal transcription level in unmethylated full mutation cell lines.

On the other hand, as in 5106 cell line, H3-K9 showed a methylation pattern similar to that of FXS cell lines. This epigenetic marker triggers the recruitment of HP1 with subsequent formation of heterochromatin. ${ }^{41}$ However, it has also been shown that heterochromatin proteins can recruit factors that facilitate the access of RNA polymerase II to heterochromatic loci. ${ }^{42}$ Therefore, it must be noted that methylated H3-K9 can also be associated with active genes. $^{43}$ Considering these findings, additional experiments are to characterize the role of HP1 at the FMR1 locus. The MECP2 protein can directly interact with the K9-specific HMT, linking histone H3-K9 methylation to histone deacetylation, independent of DNA methylation. ${ }^{44}$ An emerging view is that the methylated H3-K9 mark, which is established by the trans-acting RNAi machinery and/or DNA-binding factors, stably tethers RITS (RNA-induced transcriptional gene silencing) to chromatin, which in turn engages RDRC (RNA-dependent RNA polymerase) and perhaps Dicer to process nascent transcripts into siRNAs. ${ }^{45}$ These siRNAs feed back to trigger further recruitment of heterochromatin machinery. If and how siRNAs localize histone-modifying activities, in particular HMTs, remains to be determined. ${ }^{46,47}$ In the unmethylated full mutations we may hypothesize the presence of a defect in the RNAi machinery, causing the abnormal/absent production of siRNAs specific for FMR1 gene during early embryogenesis. It is also possible that individuals with unmethylated full mutations might have escaped a narrow time window in the course of embryogenesis, during which the epigenetic marks are established. This defect would then result in lack of recruitment of HMT and heterochromatin formation, usually observed in FXS cell lines. In fact, Eiges et $a l^{31}$ showed that the epigenetic marks (eg methylation of H3-K9) are established at an early stage of embryogenesis, whereas DNA methylation contributes to the maintenance rather than to the establishment of the silenced state of the FMR1 gene. A proposed view of the above-mentioned changes, occurring during the early stages of embryogenesis, in the presence of a CGG expansion above 200 triplets in classical FXS and in an unmethylated full mutation is reported in Figure 7.

Finally, another relevant epigenetic mark is the methylation of lysine 27 on histone H3 (H3-K27). Our results suggest a euchromatic configuration of the unmethylated full mutation, similar to that of normal control cells. Lee and Workman ${ }^{10}$ showed a concerted action of H3-K27 demethylation and H3-K4 methylation towards transcriptional activation. On the other hand, methylation of H3-K27 facilitates the binding of Polycomb to histone H3 amino-terminal tail, establishing a link between histone methylation and PcG-mediated gene silencing. ${ }^{48}$ Therefore, we could also suppose that unmethylated full mutations may be secondary to a failure of H3-K27 demethylation, causing the observed higher levels of both dimethylated H3-K27 and methylated H3-K4. It is also possible to hypothesize a mechanism of reduced function of specific H3-K27 methylating activity, with lower recruitment of Polycomb proteins responsible for gene silencing. Further experiments, focusing on these regulatory proteins, are needed to establish the molecular cause of the epigenetic marks observed in carriers of unmethylated full mutations.

In conclusion, unmethylated full mutations that we have described seem to have a remarkable structural, functional and epigenetic consistency. This is probably because of the existence of a common mechanism of origin, genetically determined. The elucidation of this mechanism is important in view of therapeutic attempts to convert a methylated to an unmethylated full mutation, restoring the expression of the FMR1 gene.

\section{Acknowledgements}

We gratefully acknowledge the financial support of Telethon Foundation (GGP06224), PRIN 2005 (Grant no. 2005060575) and Conquer Fragile $X$ Foundation to GN.

\section{References}

1 Verkerk AJ, Pieretti M, Sutcliffe JS et al: Identification of a gene (FMR-1) containing a CGG repeat coincident with a breakpoint cluster region exhibiting length variation in fragile $\mathrm{X}$ syndrome. Cell 1991; 65: 905-914.

2 Allingham-Hawkins DJ, Babul-Hirji R, Chitayat D et al: Fragile X premutation is a significant risk factor for premature ovarian failure: the International Collaborative POF in Fragile X Study: preliminary data. Am J Med Genet 1999; 83: 322-325.

3 Hagerman RJ, Leehey M, Heinrichs $\mathrm{W}$ et al: Intention tremor, parkinsonism, and generalized brain atrophy in male carriers of fragile X. Neurology 2001; 57: 127-130.

4 Tassone F, Hagerman RJ, Taylor AK, Gane LW, Godfrey TE, Hagerman PJ: Elevated levels of FMR1 mRNA in carrier males: a new mechanism of involvement in the fragile X syndrome. Am J Hum Genet 2000; 66: 6-15. 
5 Oberle' I, Rousseau F, Heitz D et al: Instability of a 550-base pair DNA segment and abnormal methylation in fragile $\mathrm{X}$ syndrome. Science 1991; 252: 1097-1102.

6 Pieretti M, Zhang F, Fu YH et al: Absence of expression of the FMR1 gene in fragile X syndrome. Cell 1991; 66: 817-822.

7 Sutcliffe JS, Nelson DL, Zhang F et al: DNA methylation represses FMR-1 transcription in fragile X syndrome. Hum Mol Genet 1992; 1: 397-400.

8 Bestor TH: The DNA methyltransferases of mammals. Hum Mol Genet 2000; 9: 2395-2402.

9 Kress C, Thomassin H, Grange T: Local DNA demethylation in vertebrates: how could it be performed and targeted? FEBS Lett 2001; 494: 135-140.

10 Lee KK, Workman JL: Histone acetyltransferase complexes: one size doesn't fit all. Nat Rev Mol Cell Biol 2007; 8: 284-295.

11 Yang XJ, Seto E: HATs and HDACs: from structure, function and regulation to novel strategies for therapy and prevention. Oncogene 2007; 26: 5310-5318.

12 Yang XJ, Seto E: Collaborative spirit of histone deacetylases in regulating chromatin structure and gene expression. Curr Opin Genet Dev 2003; 13: 143-153.

13 Zaratiegui $M$, Irvine DV, Martienssen RA: Noncoding RNAs and gene silencing. Cell 2007; 128: 763-776.

14 Lachner M, O'Sullivan RJ, Jenuwein T: An epigenetic road map for histone lysine methylation. J Cell Sci 2003; 116: 2117-2124.

15 Shi Y: Histone lysine demethylases: emerging roles in development, physiology and disease. Nat Rev 2007; 8: 829-833.

16 Shi Y, Whetstine JR: Dynamic regulation of histone lysine methylation by demethylases. Mol Cell 2007; 25: 1-14.

17 Nishioka K, Chuikov S, Sarma K et al: Set9, a novel histone H3 ethyltransferase that facilitates transcription by precluding histone tail modifications require for heterochromatin formation. Genes Dev 2002; 16: 479-489.

18 Li E: Chromatin modification and epigenetic reprogramming in mammalian development. Nat Rev Genet 2002; 3: 662-673.

19 Grewal SIS, Moazed D: Heterochromatin and epigenetic control of gene expression. Science 2003; 301: 798-802.

20 Vakoc CR, Mandat SA, Olenchock BA, Blobel GA: Histone H3 lysine 9 methylation and HP $1 \gamma$ are associated with transcription elongation through mammalian chromatin. Mol Cell 2005; 19: 381-391.

21 Yasuhara JC, Wakimoto BT: Oxymoron no more: the expanding world of heterochromatic genes. Trends Genet 2006; 22: 330-338.

22 Matzke MA, Birchler JA: RNAi-mediated pathways in the nucleus. Nature Rev Genet 2005; 6: 24-35.

23 Sparmann A, van Lohuizen M: Polycomb silencers control cell fate, development and cancer. Nature Rev Cancer 2006; 6: 846-856.

24 Lee MG, Villa R, Trojer P et al: Demethylation of H3K27 regulates polycomb recruitment and H2A ubiquitination. Science 2007; 318: $447-450$.

25 Coffee B, Zhang F, Warren S, Reines D: Acetylated histones are associated with FMR1 in normal but not fragile X syndrome cells. Nature Genet 1999; 22: 98-101.

26 Coffee B, Zhang F, Ceman S, Warren S, Reines D: Histone modifications depict an aberrantly heterochromatinized FMR1 gene in fragile X syndrome. Am J Hum Genet 2002; 71: 923-932.

27 Pietrobono R, Tabolacci E, Zalfa F et al: Molecular dissection of the events leading to inactivation of the FMR1 gene. Hum Mol Genet 2005; 14: 267-277.

28 Chiurazzi P, Pomponi MG, Pietrobono R, Bakker CE, Neri G, Oostra BA: Synergistic effect of histone hyperacetylation and DNA demethylation in the reactivation of the FMR1 gene. Hum Mol Genet 1999; 8: 2317-2323.

29 Smeets HJ, Smits AP, Verheij CE et al: Normal phenotype in two brothers with a full FMR1 mutation. Hum Mol Genet 1995; 4: 2103-2108.
30 Brouwer JR, Mientjesa EJ, Bakker CE et al: Elevated Fmr1 mRNA levels and reduced protein expression in a mouse model with an unmethylated Fragile X full mutation. Exp Cell Res 2007; 313: 244-253.

31 Eiges R, Urbach A, Malcov M et al: Developmental study of fragile $\mathrm{X}$ syndrome using human embryonic stem cells derived from preimplantation genetically diagnosed embryos. Cell Stem Cell 2007; 1: 568-577.

32 Tabolacci E, Pietrobono R, Moscato U, Oostra BA, Chiurazzi P, Neri G: Differential epigenetic modifications in the FMR1 gene of the fragile $\mathrm{X}$ syndrome after reactivating pharmacological treatments. Eur J Hum Genet 2005; 13: 641-648.

33 Pietrobono R, Pomponi MG, Tabolacci E, Oostra BA, Chiurazzi P, Neri G: Quantitative analysis of DNA demethylation and transcriptional reactivation of the FMR1 gene in fragile X cells treated with 5-azadeoxycytidine. Nucleic Acids Res 2002; 30: $3278-3285$.

34 Tabolacci E, Pomponi MG, Pietrobono R, Chiurazzi P, Neri G: A unique case of reversion to normal size of a maternal premutation FMR1 allele in a normal boy. Eur J Hum Genet 2007; 16: 209-214.

35 Wohrle D, Salat U, Hameister H, Vogel W, Steinbach P: Demethylation reactivation, and destabilization of human fragile $\mathrm{X}$ full mutation alleles in mouse embryocarcinoma cells. Am J Hum Genet 2001; 69: 504-515.

36 Tassone F, Hagerman RJ, Loesch DZ, Lachiewicz A, Taylor AK, Hagerman PJ: Fragile X males with unmethylated full mutation trinucleotide repeat expansions have elevated levels of FMR1 messenger RNA. Am J Med Genet 2000; 94: 232-236.

37 Feng Y, Zhang F, Lokey LK et al: Translational suppression by trinucleotide repeat expansion at FMR1. Science 1995; 268: 731-734.

38 Tassone F, Hagerman RJ, Taylor AK et al: Clinical involvement and protein expression in individuals with the FMR1 premutation. Am J Med Genet 2000; 91: 144-152.

39 Khateb S, Weisman-Shomer P, Hershco-Shani I, Ludwig AL, Fry M: The tetraplex $(\mathrm{CGG})_{n}$ destabilizing proteins hnRNP A2 and CBF-A enhance the in vivo translation of fragile $\mathrm{X}$ premutation mRNA. Nucleic Acids Res 2007; 35: 5775-5788.

40 Taylor AK, Tassone F, Dyer PN et al: Tissue heterogeneity of the FMR1 mutation in a high-functioning male with Fragile $\mathrm{X}$ syndrome. Am J Med Genet 1999; 84: 233-239.

41 Litt MD, Simpson M, Gaszner M, Allis CD, Felsenfeld G: Correlation between histone lysine methylation and development changes at the chicken beta-globin locus. Science 2001; 293: 2453-2455.

42 Zofall M, Grewal SI: Swi6/HP1 recruits a JmjC domain protein to facilitate transcription of heterochromatic repeats. Mol Cell 2006; 22: $681-692$.

43 Grewal SIS, Jia S: Heterochromatin revisited. Nat Rev 2007; 8: 35-46.

44 Fuks F, Hurd PJ, Wold D, Nan X, Bird AP, Kouzarides T: The methyl-CpG-binding protein MeCP2 links DNA methylation to histone methylation. J Biol Chem 2003; 278: 4035-4040.

45 Motamedi MR, Verdel A, Colmenares SU, Gerber SA, Gygi SP, Moazed D: Two RNAi complexes, RITS and RDRC, physically interact and localize to noncoding centromeric RNAs. Cell 2004; 119: $789-802$.

46 Handa V, Saha T, Usdin K: The fragile X syndrome repeats form RNA hairpins that do not activate the interferon-inducible protein kinase, PKR, but are cut by Dicer. Nucleic Acids Res 2003; 31: $6243-6248$.

47 Hong EE, Villen J, Gerace EL, Gygi SP, Moazed DA: Cullin E3 ubiquitin ligase complex associates with Rik1 and the Clr4 histone H3-K9 methyltransferase and is required for RNAi-mediated heterochromatin formation. RNA Biol 2005; 2: 106-111.

48 Cao R, Wang L, Wang H et al: Role of histone H3 lysine 27 methylation in polycomb-group silencing. Science 2002; 298: $1039-1043$.

Supplementary Information accompanies the paper on European Journal of Human Genetics website (http://www.nature.com/ejhg) 